\title{
Isolated Enteric Myeloid Sarcoma as a Rare Etiology of Small Bowel Obstruction in a Young Female Patient
}

\author{
Nagwa Abou-Ghanema, Eltaib Saad ${ }^{a}$, , Ira A. Oliff ${ }^{b}$, \\ Adi Gidron $^{\mathrm{b}}$, Dorota Filipiuk ${ }^{\mathrm{c}}$
}

\begin{abstract}
Myeloid sarcoma (MS) is an extra-medullary solid tumor consisting of myeloid blasts or immature myeloid cells. MS is usually associated with acute myeloid leukemia (AML) and other myeloproliferative neoplasms or myelodysplastic disorders. Isolated MS is a rare clinical entity, and the small bowel is a rare phenomenon for the occurrence of MS. A 30-year-old African American female patient with a past medical history of asthma presented with acute abdominal pain and vomiting for 3 days. Imaging revealed small bowel obstruction with a transition point at a suspicious mass in the distal ileum mimicking carcinoid tumors. She underwent an uneventful laparoscopic resection of this mass with primary bowel anastomosis. Histopathology of the resected mass revealed immature myeloid cells that stained positive for myeloperoxidase and CD34/CD117, in keeping with a small bowel MS. A bone marrow examination was negative for concurrent AML. Cytogenetic analysis revealed MYH11/CBFB fusion and an inversion 16 chromosomal aberration which are rarely associated with myeloid disorders. The patient was commenced on systemic chemotherapy to achieve remission and prevent progression to AML. The literature is reviewed, and all cases of small bowel MS are presented in this report. Non-leukemic small bowel MS is an exceptional presentation. We described a case of isolated enteric MS, which was associated with a rare MYH11/CBFB fusion and inversion 16 chromosomal aberration. The diagnosis of small bowel MS can be extremely challenging due to the rarity of the disease and non-specific nature of clinical and radiological features. A histopathological examination with immunohistochemistry staining is imperative to establish an accurate diagnosis. Isolated small bowel MS deserves special attention as it warrants systemic chemotherapy to prevent transformation into AML.
\end{abstract}

Keywords: Myeloid sarcoma; Acute myeloid leukemia; Small bowel obstruction; Rare etiology; Chemotherapy

Manuscript submitted November 26, 2021, accepted December 30, 2021

Published online January 10, 2022

${ }^{a}$ Department of Internal Medicine, Saint Francis Presence Hospital, Evanston, IL, USA

bDepartment of Oncology, Saint Francis Presence Hospital, Evanston, IL, USA 'Department of Pathology, Saint Francis Presence Hospital, Evanston, IL, USA

${ }^{\mathrm{d}}$ Corresponding Author: Eltaib Saad, Department of Internal Medicine, Saint Francis Presence Hospital, Evanston, IL, USA.

Email: Eltaib.Saad@amitahealth.org

doi: https://doi.org/10.14740/gr1481

\section{Introduction}

Myeloid sarcoma (MS), also known as granulocytic sarcoma, chloroma, and myeloblastoma, is an extra-medullary solid tumor consisting of myeloid blasts originating from one or more lineages [1-3]. MS is usually associated with acute myeloid leukemia (AML) and other myelodysplastic disorders [1-3]. Isolated or non-leukemic MS is a rare clinical entity [1-5], and it tends to occur in the subperiosteal bones, skin, and lymphoid organs $[4,5]$. The small bowel is an unusual site for the occurrence of MS [6-9].

Herein, we describe a rare case of primary enteric MS, which presented clinically as acute small bowel obstruction in a young female patient. The radiological appearances of the obstructing distal ileal mass simulated carcinoid tumors on imaging. The diagnosis was established by histopathological examination and immunohistochemistry staining of the resected mass. The patient was commenced on systemic AML-type chemotherapy to achieve remission. The current literature is reviewed, and all reported cases of small bowel MS in the English medical literature are presented in this report.

\section{Case Report}

A 32-year-old African American female patient presented to our hospital with acute lower abdominal pain and non-bilious vomiting that started 3 days prior to her presentation. The pain was in the suprapubic area and radiated up to right lower quadrant. Review of systems was pertinently negative for abnormal bowel movements and B-symptoms including fever, chills, and rash. Her pain did not improve despite oral and intravenous analgesics. Past medical history is significant for well-controlled asthma on as needed albuterol inhaler and montelukast. She has no surgical history and no allergies. She does not smoke or use illicit drugs. On physical examination, the patient appeared in moderate distress however vitally stable with a blood pressure of 113/72 $\mathrm{mm} \mathrm{Hg}$, and a heart rate of 72 beats per minute. Abdominal examination was notable for generalized abdominal tenderness in epigastric and umbilical area along with the right lumbar region. The rest of the examination was essentially unremarkable. Complete blood count was normal. Electrolytes, renal function, and liver functions were normal. Urinalysis was unremarkable. Urine pregnancy test was negative.

A computer tomography (CT) of the abdomen demon- 

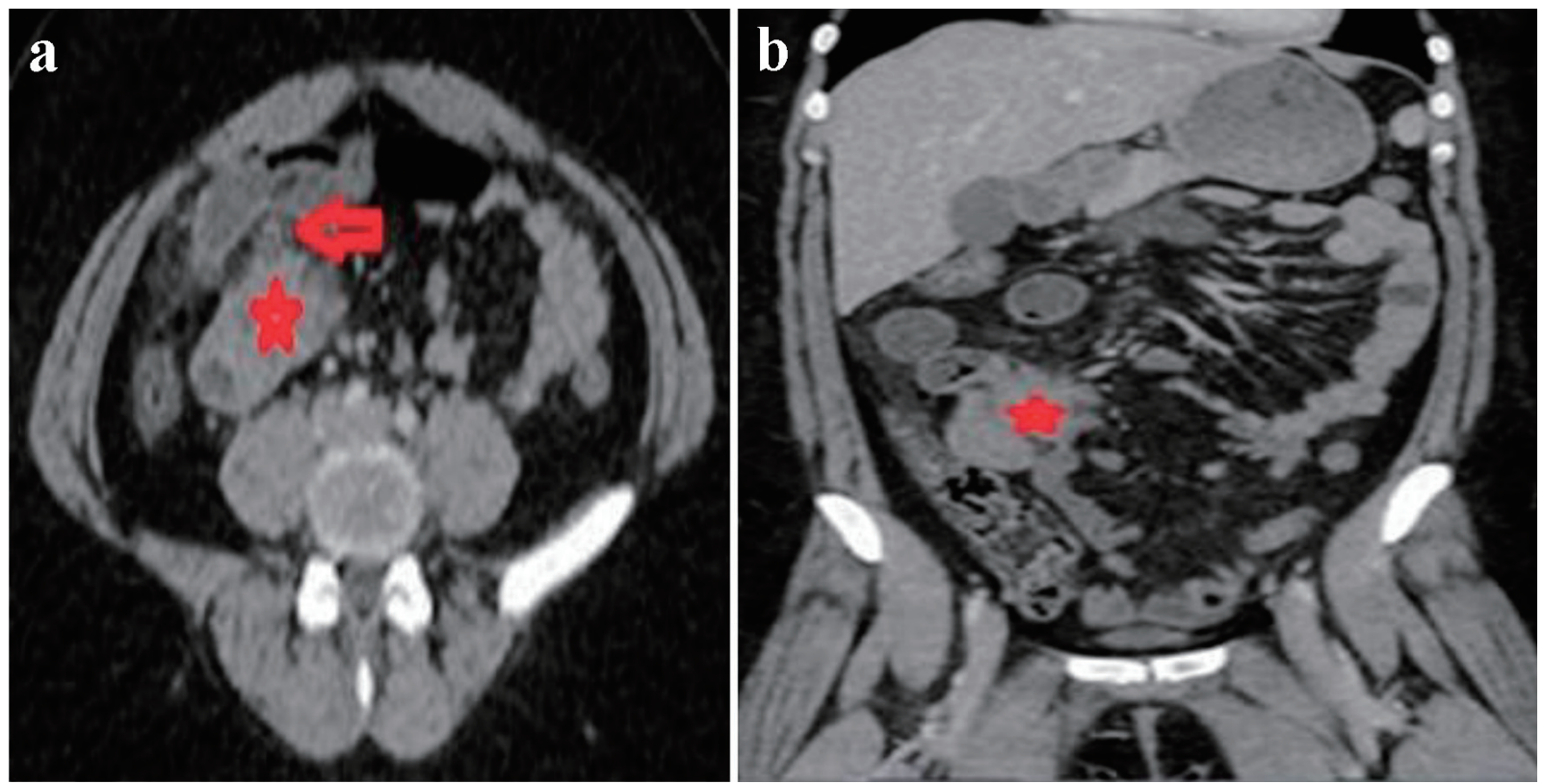

Figure 1. Axial (a) and coronal (b) images of CT of abdomen revealing mildly dilated small bowel loops with a transition point to the right of the midline (red arrow in a) in an area of a spiculated mass in the distal ileum simulating carcinoid tumors (red stars in a and b). СT: computed tomography.

strated a small bowel obstruction with dilated small loops of bowel with a transition point on the right of the mid-abdominal region at a spiculated mass arising in the distal ileum, measuring about $4.0 \times 3.9 \times 4.4 \mathrm{~cm}$, along with mild fat stranding and local inflammatory changes, simulating small bowel carcinoid tumors (Fig. 1a, b). Patient was admitted for small bowel obstruction along with intractable pain and was urgently taken for an exploratory laparoscopy. A $3 \times 3 \mathrm{~cm}$ mass was visualized in the distal ileum adhering to the associated mesentery, with an edematous and puckered look. The mass was resected with primary bowel anastomosis, and the postoperative course was uneventful.

Histopathological examination of the resected mass revealed a transmural bowel wall infiltration with a dense population of polymorphous medium and large-sized immature myeloid cells with scant eosinophilic cytoplasm (Fig. 2a-c) that stained positive for myeloperoxidase and CD34/CD117 stains, consistent with a small bowel MS (Fig. 3a, b). A bone marrow biopsy was performed to rule out concurrent AML, which revealed normocellular with intact tri-lineage hematopoiesis, without evidence of acute myeloid or myeloproliferative disorders. Cytogenetics studies with fluorescence in situ hybridization (FISH) revealed that approximately $80 \%$ of nuclei had MYH11/CBFB fusion and an inv(16) chromosomal aberration. Staging imaging with positron emission tomography with 18-fluorodeoxyglucose imaging (PET-FDG) revealed no evidence for local, regional, and distant metastasis and patient was referred to oncology department.

Patient was commended on systemic chemotherapy to achieve remission and prevent progression into AML, and she was considered for bone marrow transplantation (BMT) if complete remission is achieved.
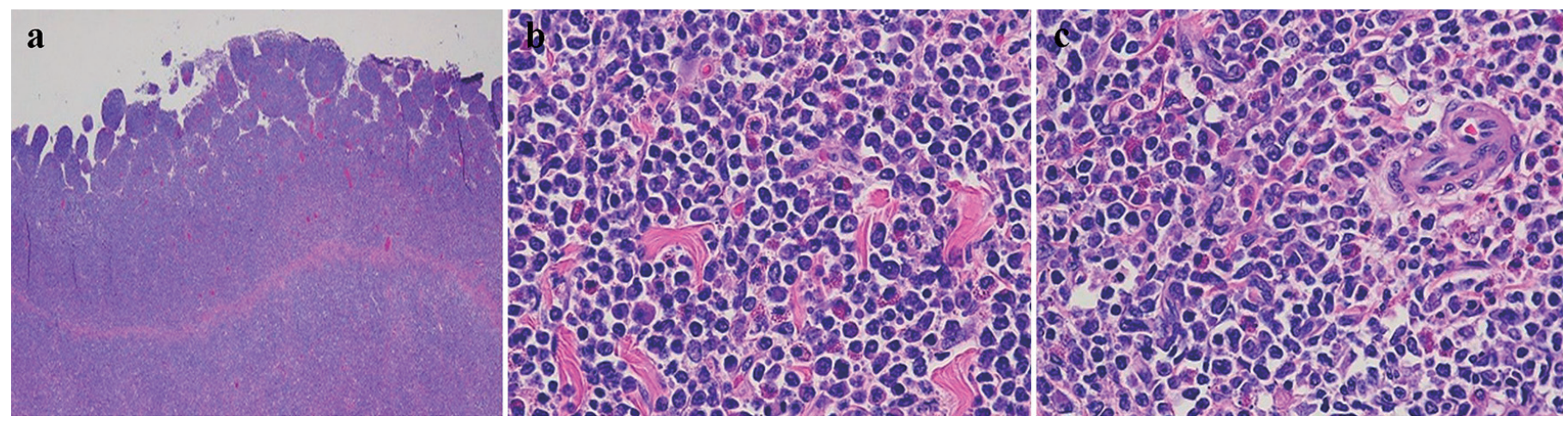

Figure 2. Hematoxylin and eosin-stained sections of the small bowel mass. (a) Diffuse transmural infiltration with a dense population of polymorphous medium and large-sized immature cells with scant eosinophilic cytoplasm $(\times 2)$. (b, c) Immature neoplastic cells with rounded hyperchromatic nuclei and scattered eosinophils $(\times 200)$. 

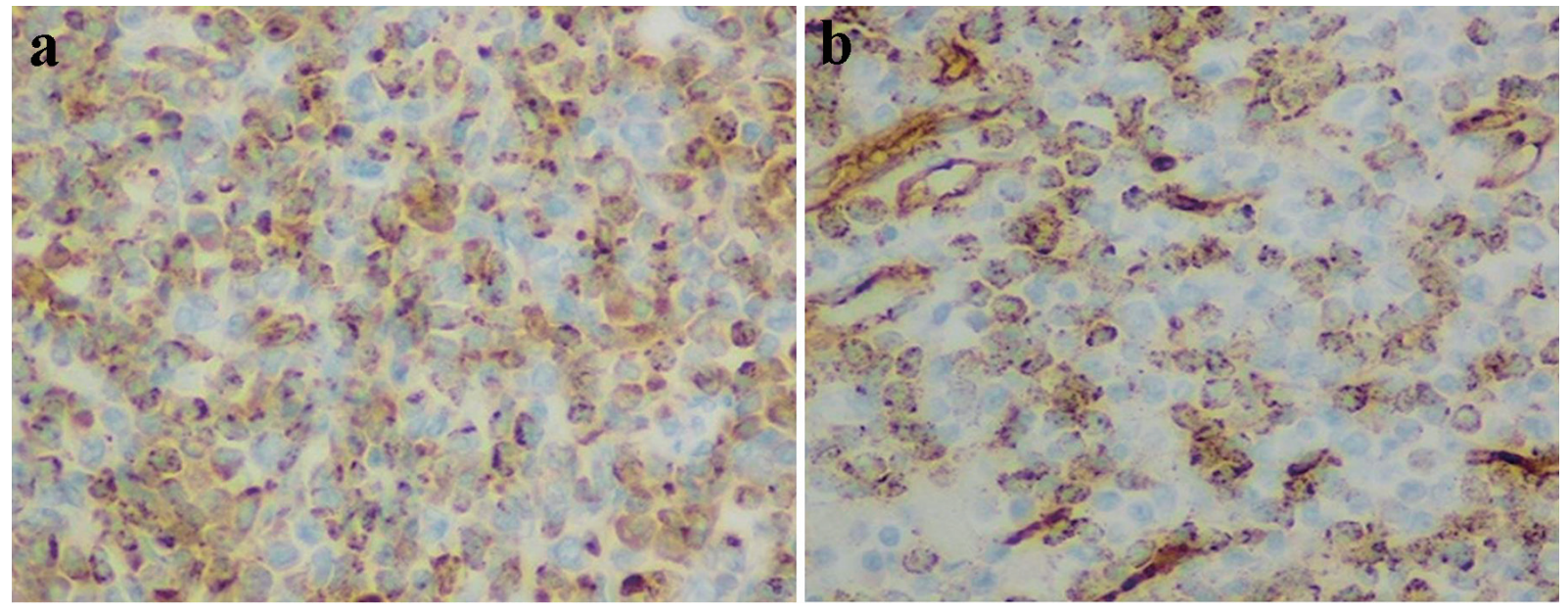

Figure 3. Immunohistochemistry staining of myeloid cells (× 200). Positive staining with myeloperoxidase (a) and CD34 (b).

\section{Discussion}

Isolated MS is a rare clinical entity, wherein only $1.4-9.0 \%$ of all patients with AML are reported to develop MS [1, 2]. MS can present variably in relation to leukemia and myelodysplastic disorders: 1) MS concurrent with leukemia; 2) MS as a harbinger of leukemia; 3) MS concurrent with other myelodysplastic syndromes; and 4) isolated MS in a patient with no leukemia or myelodysplasia [7-9]. MS can represent an initial manifestation of an associated AML but may also be a culprit of AML relapse or recurrence [8]. The most reported sites of the occurrence of MS are subperiosteal bone, skin, and lymph nodes $[4,5]$. MS in the small bowel is quite uncommon; only $6.5 \%$ of MS cases have been found to occur in the gut [9]. Therefore, isolated MS of the small bowel in our non-leukemic patient represents an oncological rarity [10-12].

As per our review of the available English medical literature, we identified a total of 26 cases of small bowel MS [1-26]. These reviewed cases all involved MS specifically in the small bowel. Table 1 summarizes the demographic characteristic of reported cases, clinical presentation, anatomical site and regional disease extent, association with AML, and the management offered for each patient with the outcome at the time of publication of these reports. The male to female ratio was 2:1, and the age ranged considerably from 13 to 65 years. Abdominal pain, vomiting, and constipation were the chief presenting complaints. Interestingly, one patient presented primarily with bloody diarrhea due to ulcerative ileal lesions [17]. Duration of symptoms varied widely from a few days (as in our patient) to several months $[7,21]$. The distal ileum was the most involved site ( $>40 \%$ of reported cases), and this finding may be attributable to abundance of Peyer's patches in the distal ileum [23]. Six patients had concurrent AML $[3,4,7,17,21,25]$, and leukemic status was unspecified in two patients.

Imaging features included focal bowel wall thickening, polypoidal mass with intraluminal or exophytic parts or both [2]. The lesions are usually unifocal, but multifocal involvement was also reported [8]. Additionally, two cases masqueraded radiologically as small bowel intussusception $[3,11]$. Extensive omental and mesenteric infiltration with regional lymphadenopathy was a common finding as well [8, $15,16,19,20,25]$. Nevertheless, these imaging appearances are rather non-specific, and MS can almost be indistinguishable on imaging from small bowel non-Hodgkin lymphomas, small round-cell tumors such as neuroendocrine tumors and carcinoid tumors (as in our case), and small bowel metastatic cancers [13].

Histopathological examination with immunohistochemistry is the gold standard to establish MS diagnosis and exclude other differentials [3, 4]. Pathological findings of MS may simulate a range of benign and neoplastic tumors including gastrointestinal stromal tumors (GISTs), malignant lymphoma, melanoma, and undifferentiated tumors such as Ewing's sarcoma [24]. Immunohistochemical panel for myeloid markers, like MPO, CD34/CD 117, and CD68 stains, should help in further differentiation [24]. Cytogenetic analysis demonstrated an inv(16) chromosomal anomaly, which is rarely associated with MS and actually tends to present in AML; there were three cases in our review of enteric MS cases that were associated with this inversion $[3,25,26]$. However, in contrast to the first two cases $[3,25]$, our case and a similar one [26] describe an inv(16) aberration in a non-leukemic patient with small bowel MS, which represents an even rarer combination. Furthermore, CBFB/MYH11 fusion detected in our patient has been shown to demonstrate a predilection for an abdominal location of MS [16, 25, 26]. While the latter fusion has a favorable prognosis in AML, the prognostic significance in MS is less well-defined [27], and further studies are warranted to evaluate for its potential prognostic value in MS population.

Most enteric MS patients underwent surgical resection as they chiefly presented with bowel obstruction, in keeping with our case. The majority of patients were offered AML-type chemotherapy and they achieved complete remission. One patient opted for active surveillance of AML [18]. Another one developed relapsing small bowel MS and AML within 3 months of the original MS diagnosis, after initially declining 


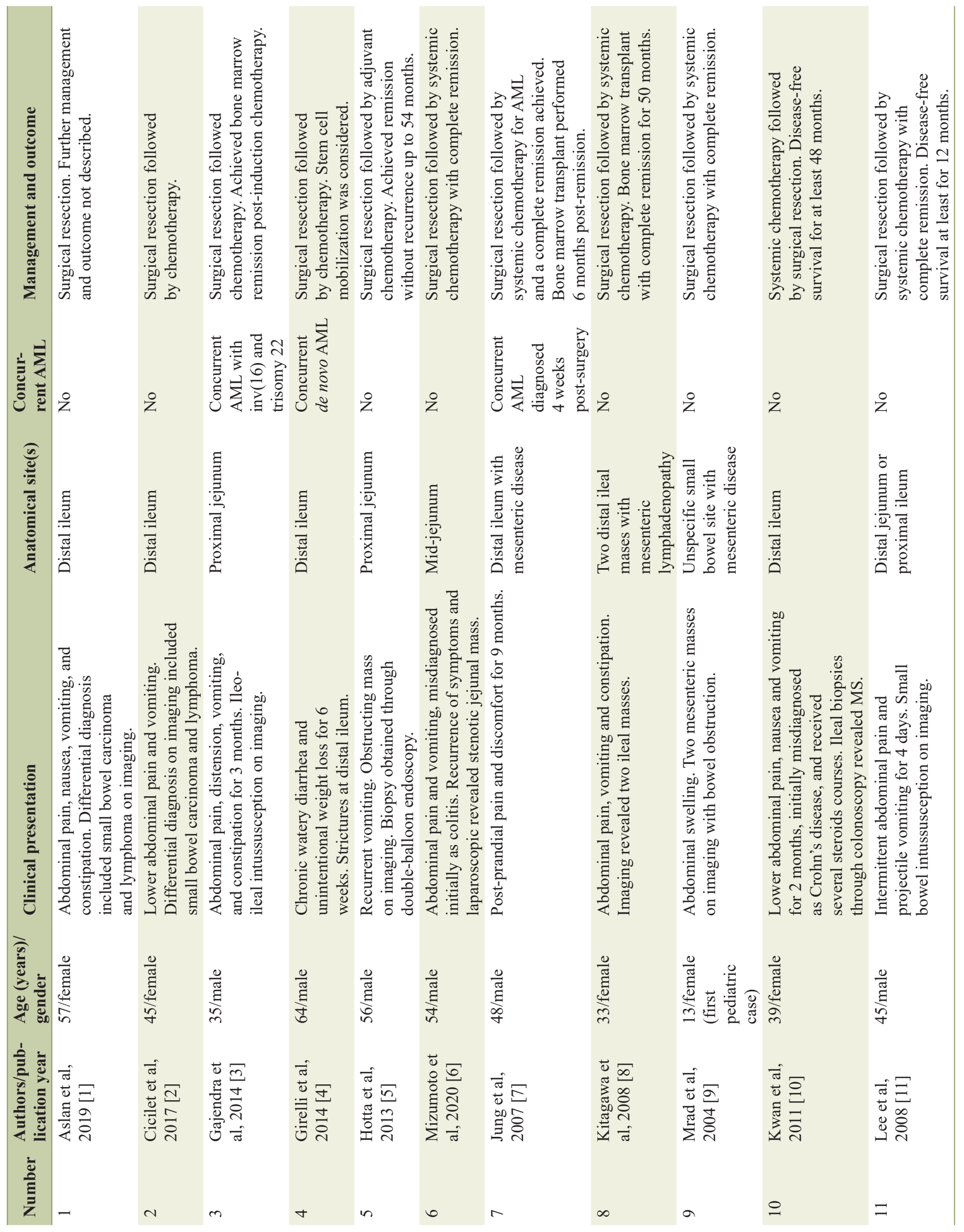




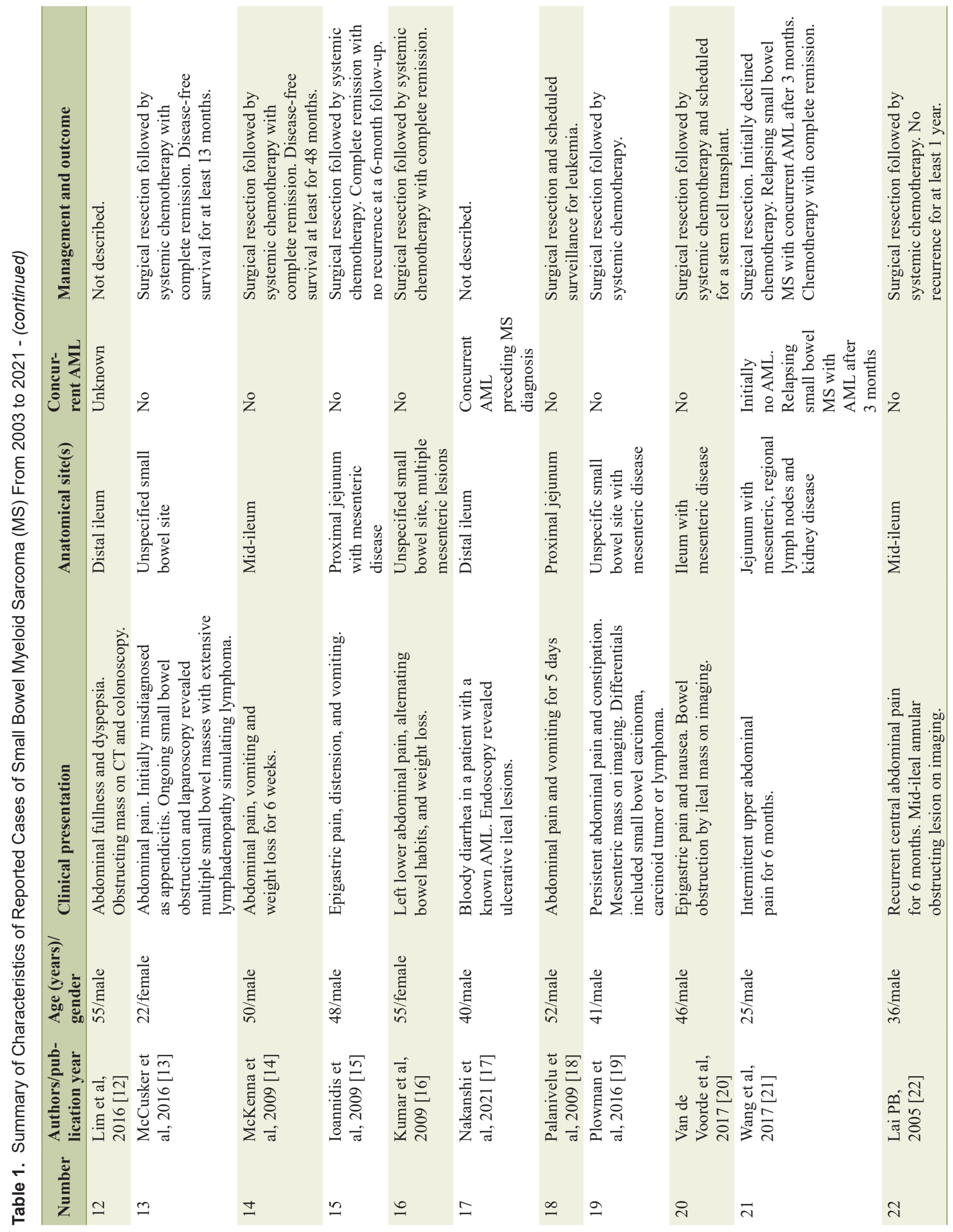




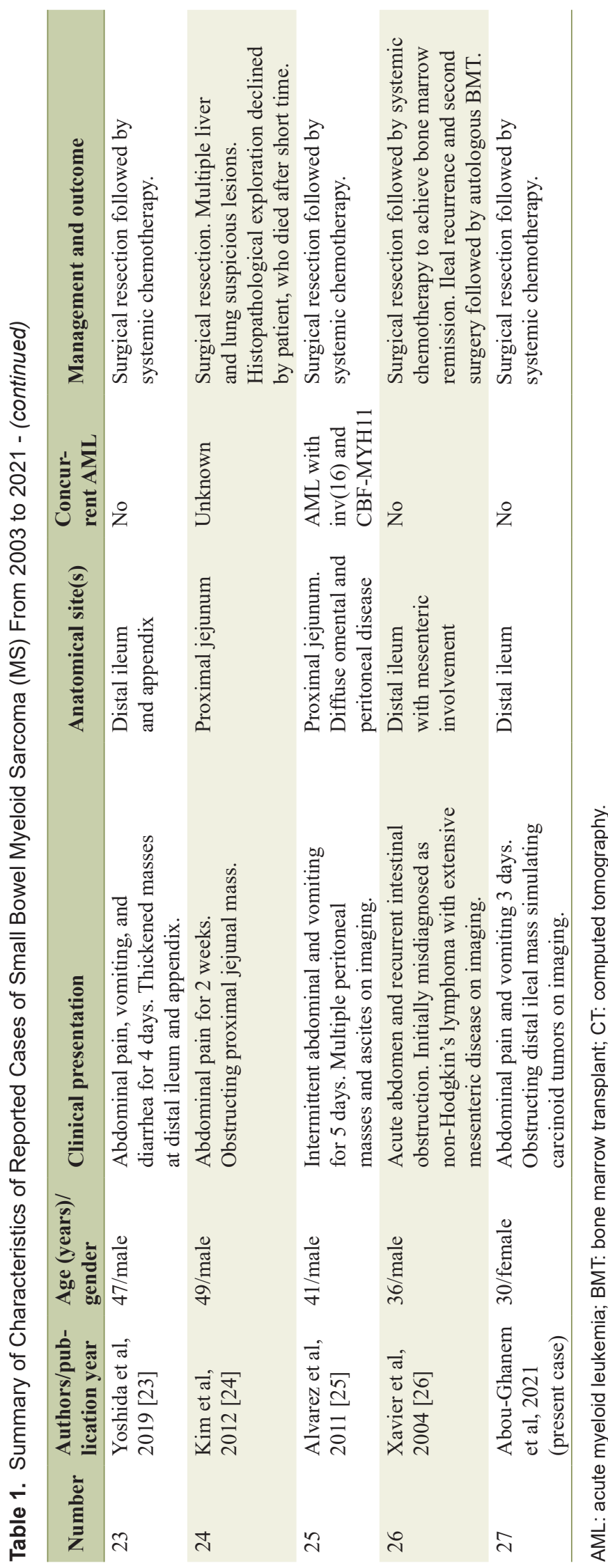

adjuvant chemotherapy, before achieving complete remission after receiving adjuvant chemotherapy [21]. In fact, systemic chemotherapy has been associated with reduced risks of AML development, prolonged non-leukemic phase, and improved disease-free survival [8]. Nevertheless, no systematic reviews have yet evaluated the long-term outcome and prognosis of the small bowel MS as a definite clinical entity, and most of current data were derived primarily from isolated case reports. Furthermore, three patients underwent post-remission BMT $[7,8]$ and two others were considered for the latter following chemotherapy-achieved remission [4, 20, 26]. Early reports have shown a better outcome of MS patients who were offered BMT [8].

\section{Conclusion}

Non-leukemic small bowel MS is an exceedingly rare entity. We describe a case of primary enteric MS as an unusual etiology of small bowel obstruction in a young female patient. The preoperative diagnosis of primary small bowel MS can be extremely challenging, as demonstrated in our cases, due to the rarity of the disease and non-specific nature of clinical and radiological features. In fact, this is the second case in literature of isolated small bowel MS where cytogenic findings of MYH11/CBFB fusion and an inversion (16) chromosomal aberration are described. Isolated small bowel MS deserves special attention as it warrants systemic chemotherapy to prevent development of AML and improve disease-free survival.

\section{Learning objectives}

Non-leukemic small bowel MS is an exceedingly rare entity that deserves special attention as it stimulates a range of inflammatory and neoplastic small bowel conditions both clinically and radiologically. Therefore, surgeons and gastroenterologists should be aware of this clinical phenomenon despite being a rare occurrence.

The diagnosis of enteric MS is highly challenging due to the disease's rarity and lack of clinical and radiological features. Histopathology examination with immunohistochemistry analysis is imperative to making the definitive diagnosis.

Cytogenetic studies should be employed in all cases of enteric MS as they may have a potential prognostic value. Further research should focus on the prognostic benefits of CBFB/ MYH11 fusion in enteric MS population.

Systemic chemotherapy is warranted in non-leukemic enteric MS so as to achieve remission, prevent progression to $\mathrm{AML}$, and prolong disease-free intervals.

\section{Acknowledgments}

Authors would like to acknowledge the Department of Pathology at Northwestern University Hospital for providing Pathology and Immunohistochemistry images for review. 


\section{Financial Disclosure}

None to declare.

\section{Conflict of Interest}

The authors declare that no conflict of interest regarding the publication of this case report.

\section{Informed Consent}

Informed written consent was obtained from the patient to write and publish their case as a case report with all accompanying clinical and radiological images. No identifying information has been used in this article.

\section{Author Contributions}

NA and ES contributed equally to conceptualization, designing of the report and writing first manuscript. IO and $A G$ performed critical review and contributed to final manuscript editing. DF provided pathology and immunohistochemistry images and contributed to manuscript writing. All authors reviewed the last draft and agreed for submission.

\section{Data Availability}

The authors declare that data supporting the findings of this study are available within the article.

\section{References}

1. Aslan B, Tuney D, Ercetin Y, Bozkurt SU, Uprak TK. De novo myeloid sarcoma as a rare cause of small bowel obstruction: CT findings and histopathologic correlation. Radiol Case Rep. 2019;14(12):1487-1490.

2. Cicilet S, Tom FK, Philip B, Biswas A. Primary myeloid sarcoma of small bowel. BMJ Case Rep. 2017;2017:bcr2017.

3. Gajendra S, Gogia A, Das P, Gupta R, Tanwar P. Acute myeloid leukemia presenting as "bowel upset": a case report. J Clin Diagn Res. 2014;8(7):FD09-10.

4. Girelli CM, Carsenzuola V, Latargia M, Aguzzi A, Serio G. Small-bowel myeloid sarcoma: Report of a case with atypical presentation. Int J Surg Case Rep. 2014;5(9):613616.

5. Hotta K, Kunieda K. Granulocytic sarcoma of the jejunum diagnosed by biopsies during double-balloon endoscopy before treatment (with video). Dig Endosc. 2013; 25(4):468.

6. Mizumoto R, Tsujie M, Wakasa T, Kitani K, Manabe H, Fukuda S, Okada K, et al. Isolated myeloid sarcoma presenting with small bowel obstruction: a case report. Surg
Case Rep. 2020;6(1):2.

7. Jung SH, Kim HC, Yu CS, Kim JC. Solitary preleukemic granulocytic sarcoma as a cause of small bowel obstruction. Gut Liver. 2007;1(1):82-86.

8. Kitagawa Y, Sameshima Y, Shiozaki H, Ogawa S, Masuda A, Mori SI, Teramura M, et al. Isolated granulocytic sarcoma of the small intestine successfully treated with chemotherapy and bone marrow transplantation. Int J Hematol. 2008;87(4):410-413.

9. Mrad K, Abid L, Driss M, Ben Abid H, Ben Romdhane K. Granulocytic sarcoma of the small intestine in a child without leukemia: report of a case with cytologic findings and immunophenotyping pitfalls. Acta Cytol. 2004;48(5):641-644.

10. Kwan LY, Targan SR, Shih DQ. A case of steroid-dependent myeloid granulocytic sarcoma masquerading as Crohn's disease. World J Gastroenterol. 2011;17(19):2446-2449.

11. Lee SY, Park SJ, Kim YH, Lee JH. Nonleukemic granulocytic sarcoma presenting as intussusception of small bowel. Int J Clin Oncol. 2008;13(5):467-470.

12. Lim SW, Lee HL, Lee KN, Jun DW, Kim IY, Kim E, Ahn $\mathrm{H}$, et al. A case of myeloid sarcoma of intestine. Korean J Gastroenterol. 2016;68(3):148-151.

13. McCusker S, Trangucci J, Frederick W, Richi AA, Abunnaja S. Primary Myeloid Sarcoma of the Small Intestine: Case Report and Literature Review. Conn Med. 2016;80(6):349-352.

14. McKenna M, Arnold C, Catherwood MA, Humphreys MW, Cuthbert RJ, Bueso-Ramos C, McManus DT. Myeloid sarcoma of the small bowel associated with a CBFbeta/MYH11 fusion and $\operatorname{inv}(16)(p 13 q 22)$ : a case report. J Clin Pathol. 2009;62(8):757-759.

15. Ioannidis O, Cheva A, Kakoutis E, Rafail S, Kotronis A, Chatzopoulos S, Makrantonakis N. Primary myeloid sarcoma of the jejunum and greater omentum causing small intestine obstruction. Acta Gastroenterol Belg. 2009;72(3):369-372.

16. Kumar B, Bommana V, Irani F, Kasmani R, Mian A, Mahajan K. An uncommon cause of small bowel obstruction: isolated primary granulocytic sarcoma. QJM. 2009;102(7):491-493.

17. Nakanishi R, Henmi S, Marusawa H. Small bowel bleeding caused by myeloid sarcoma. Intern Med. 2022;61(1):123-124.

18. Palanivelu C, Rangarajan M, Senthilkumar R, Annapoorni S. Laparoscopic management of an obstructing granulocytic sarcoma of the jejunum causing intussusception in a nonleukemic patient: report of a case. Surg Today. 2009;39(7):606-609.

19. Plowman RS, Nguyen BD. Gastrointestinal: Small bowel and mesenteric primary myeloid sarcoma: PET/CT imaging. J Gastroenterol Hepatol. 2016;31(5):907.

20. Van de Voorde N, Min W, Salgado R. De novo myeloid sarcoma as a cause of small bowel obstruction: a case report. J Belg Soc Radiol. 2017;101(1):28.

21. Wang P, Li Q, Zhang L, Ji H, Zhang CZ, Wang B. A myeloid sarcoma involving the small intestine, kidneys, mesentery, and mesenteric lymph nodes: A case report and literature review. Medicine (Baltimore). 
2017;96(42):e7934.

22. Wong SW, Lai CK, Lee KF, Lai PB. Granulocytic sarcoma of the small bowel causing intestinal obstruction. Hong Kong Med J. 2005;11(3):204-206.

23. Yoshida M, Ogami T, Morgenstern N, Du L. Myeloid sarcoma resulting in a small bowel obstruction with multiple site involvement including ileum and appendix. J Surg Case Rep. 2019;2019(9):rjz248.

24. Kim NR, Lee WK, Lee JI, Cho HY. Multiple jejunal myeloid sarcomas presenting with intestinal obstruction in a non-leukemic patient: a case report with ultrastructural observations. Korean J Pathol. 2012;46(6):590-594.

25. Alvarez P, Navascues CA, Ordieres C, Pipa M, Vega IF,
Granero P, Alvarez JA, et al. Granulocytic sarcoma of the small bowel, greater omentum and peritoneum associated with a CBFbeta/MYH11 fusion and inv(16) (p13q22): a case report. Int Arch Med. 2011;4(1):3.

26. Xavier SG, Fagundes EM, Hassan R, Bacchi C, Conchon M, Tabak DG, Spector N, et al. Granulocytic sarcoma of the small intestine with CBFbeta/MYH11 fusion gene: report of an aleukaemic case and review of the literature. Leuk Res. 2003;27(11):1063-1066.

27. Swerdlow SH, Campo E, Harris NL. Mature T and NK cell neoplasms. In: WHO classification of Tumours of Haematopoietic and Lymphoid Tissues. Revised 2017. IARC Press, Lyon, France. p. 346-422. 